Annals of Pure and Applied Mathematics

Vol. 21, No. 1, 2020, 1-14

ISSN: 2279-087X (P), 2279-0888(online)

Published on 31 January 2020

www.researchmathsci.org

DOI: http://dx.doi.org/10.22457/apam.v21n1a1645

Annals of

Pure and Applied

Mathematics

\title{
On Zagreb Indices of Graphs with a Deleted Edge
}

\author{
Mahesh Kale $^{1}$ and Minirani S. ${ }^{2}$ \\ ${ }^{1}$ Department of Basic Sciences and Humanities, MPSTME \\ SVKM's NMIMS Deemed to be University, Mumbai, India \\ 12E-mail: mnk.maths@ gmail.com; ${ }^{2}$ E-mail: miniranis@yahoo.com \\ ${ }^{1}$ Corresponding author
}

Received 31 December 2019; accepted 29 January 2020

\begin{abstract}
In the fields of chemical graph theory, mathematical chemistry, pharmaceuticals, topological indices are calculated based on the molecular graph of a chemical compound. Topological indices are used in various fields such as in the developments of Quantitative Structure Activity Relationships (QSARs) and Quantitative Structure-Property Relationships (QSPRs). In Chemical Science, to study the physiochemical properties of molecules most commonly used indices are Zagreb indices. In this paper, we discuss the effect of an edge deletion on Zagreb indices of simple graphs, also we verify the results obtained on some standard simple graphs such as $P_{n}, C_{n}, S_{n}, K_{n}$ and $K_{m, n}$.
\end{abstract}

Keywords: Zagreb indices, Multiplicative Zagreb indices, Hyper Zagreb index, Multiplicative Hyper Zagreb indices, Forgotten topological index

AMS Mathematics Subject Classification (2010): 05C05, 05C07, 05 C90

\section{Introduction}

In 1972 [1], Gutman introduced the first Zagreb index, which is among the oldest topological index. Molecular graphs are graphical models of molecules in which atoms and chemical bonds are represented by vertices and edges respectively. Many graph invariants and their applications have been studied by graph theorists and chemists. The physio-chemical properties of molecules can be studied with the help of different topological indices such as Zagreb indices. It gives an important molecular descriptor and it has been correlated with many chemical properties. Mathematical properties and applications of Zagreb multiplicative indices are discussed in $[2,3,4,5,6,7,8,9,10,11$, 12, 13, 14]. In [15], Furtula and Gutman re-introduced the forgotten topological index. The extension of studies on topological indices to fuzzy graphs can be found in $[16,17]$.

Let $\mathrm{G}$ be a connected, undirected and finite simple graph with vertex set $\mathrm{V}$ and edge set E. If $u \in V$ then we denote all adjacent vertices to u by $N(u)$ and cardinality of $N(u)$ by $d_{u}$, which is called the degree of vertex $u$. A vertex with degree one is called pendent vertex and an edge connecting pendent vertex is called a pendent edge. Graphs 
Mahesh Kale and Minirani S.

$P_{n}, C_{n}, S_{n}, K_{n}, K_{m, n}$ denotes path, cycle, star, complete and complete-bipartite graphs respectively.

A chemical structure can be represented using a graph commonly known as a molecular graph or a chemical graph. In molecular graphs, atoms of a structure correspond to vertices and the bonds to edges of the graph. The topological and topographical character of the structural information of the activity characterizes structure-activity problems in the chemical field. Traditional statistical methodology requires this predictive information to be mapped to a vector space. To circumvent this vexing conversion of structural information to vector form, the edge deletion metric is required on a space of chemical graphs that defines the topology of molecules. Here we discuss the Zagreb indices and the effect of edge deletion on this graph invariant.

\section{Preliminaries}

In this section, we recall some definitions of Zagreb indices, which will play an important role in the subsequent proofs.

Zagreb first and second indices of simple graph $\mathrm{G}$ are defined as

$$
M_{1}(G)=\sum_{u v \in E}\left[d_{u}+d_{v}\right]=\sum_{u \in V} d_{u}^{2}, M_{2}(G)=\sum_{u v \in E} d_{u} \cdot d_{v}
$$

Authors [18], in 2010 introduced the first and second multiplicative Zagreb indices respectively denoted by $\Pi_{1}(G), \Pi_{2}(G)$ and are defined as

$$
\Pi_{1}(G)=\prod_{u \in V} d_{u}^{2}, \Pi_{2}(G)=\prod_{u v E} d_{u} \cdot d_{v}
$$

Eliasi [19], in 2012 introduced another multiplicative-sum version of Zagreb index denoted by $\Pi_{1}^{*}(G)$ and defined as

$$
\Pi_{1}^{*}(G)=\prod_{u v \in E}[d u+d v]
$$

In general $\Pi_{1} \leq \Pi_{1}^{*}(G)$. In fact $\Pi_{1}=\Pi_{1}^{*}(G)$ only if $\mathrm{G}$ is a cycle.

Gutman in [20] have defined the second Zagreb multiplicative index alternatively as

$$
\Pi_{2}(G)=\prod_{u \in V}\left(d_{u}\right)^{d_{u}}
$$

Recently, authors in [21] proposed new invariant Hyper Zagreb index, defined as

$$
H M(G)=\sum_{u v \in E}\left(d_{u}+d_{v}\right)^{2}
$$

Later, Kulli [22] introduced the multiplicative Hyper Zagreb indices which are defined as

$$
H \Pi_{1}(G)=\prod_{u v E}\left[d_{u}+d_{v}\right]^{2} \text { and } H \Pi_{2}(G)=\prod_{u v \in E}\left[d_{u}^{2} \cdot d_{v}^{2}\right]
$$

where $d_{u}$ denotes the degree of vertex 'u'.

In [15], Furtula and Gutman re-introduced Forgotten topological index, defined as

$$
F(G)=\sum_{u \in V}\left[d_{u}\right]^{3}=\prod_{u v E}\left[d_{u}^{2}+d_{v}^{2}\right]
$$

In the following section we put forward some important results on the effect of edge deletion on Zagreb indices. Also we discuss the effect in some standard graphs such as $P_{n}, C_{n}, S_{n}, K_{n}$ and $K_{m, n}$. 
On Zagreb Indices of Graphs with a Deleted Edge

\section{Main Results}

In this section, we study the effect on Zagreb indices, after deleting an edge from a simple graph $G$.

Theorem 3.1. Let $G(V, E)$ be a simple graph, $V=\left\{u_{1}, u_{2}, \ldots, u_{n}\right\}$. Let $e \in E$ be an edge incident to vertices $u_{1}$ and $u_{2}$ of graph $\mathrm{G}$, then the effect of edge deletion on the first Zagreb index is given by

$$
M_{1}(G-\{e\})=M_{1}(G)-2\left[d_{u_{1}}+d_{u_{2}}-1\right]
$$

Proof: By definition, first Zagreb index is given by

$$
M_{1}(G)=\sum_{u \in V} d_{u}^{2}=d_{1}^{2}+d_{2}^{2}+\ldots+d_{u_{1}}^{2}+d_{u_{2}}^{2}+\ldots+d_{u_{n}}^{2}
$$

If $e=\left(u_{1}, u_{2}\right)$ is deleted then

$$
\begin{gathered}
M_{1}(G-\{e\})=d_{1}^{2}+d_{2}^{2}+\ldots+\left(d_{u_{1}}-1\right)^{2}+\left(d_{u_{2}}-1\right)^{2}+\ldots+d_{u_{n}}^{2} \\
M_{1}(G-\{e\})-M_{1}(G)=\left(d_{u_{1}}-1\right)^{2}+\left(d_{u_{2}}-1\right)^{2}-d_{u_{1}}^{2}-d_{u_{2}}^{2} \\
M_{1}(G-\{e\})=M_{1}(G)-2\left(d_{u_{1}}+d_{u_{2}}-1\right)
\end{gathered}
$$

Theorem 3.2. Let $G(V, E)$ be a simple graph, with $|V|=n$ and $|E|=m$. For simplicity, as the labeling of vertices is not important, let $e \in E$ be an edge incident to vertices $u_{1}$ and $u_{2}$ of the graph $G$. Let $N\left(u_{1}\right)=\left\{u_{2}, v_{1}, v_{2}, \ldots, v_{k}\right\}$ and $N\left(u_{2}\right)=\left\{u_{1}, w_{1}, w_{2}, \ldots, w_{l}\right\}$, where $k+l+2 \leq n$, then effect of edge deletion on the second Zagreb index is given by

$$
M_{2}(G-\{e\})=M_{2}(G)-\sum_{i=1}^{k} d_{v_{i}}-\sum_{j=1}^{l} d_{w_{j}}-d_{u_{1}} \cdot d_{u_{2}}
$$

Proof: By definition, second Zagreb index is given by

$$
\begin{aligned}
M_{2}(G)=\sum_{u v \in E} d_{u} \cdot d_{v}= & d_{u_{1}} \cdot d_{u_{2}}+\left[d_{v_{1}} \cdot d_{u_{1}}+d_{v_{2}} \cdot d_{u_{1}}+\ldots+d_{v_{k}} \cdot d_{u_{1}}\right] \\
& +\left[d_{w_{1}} \cdot d_{u_{2}}+d_{w_{2}} \cdot d_{u_{2}}+\ldots+d_{w_{l}} \cdot d_{u_{2}}\right]+\mathcal{P}
\end{aligned}
$$

where $\mathcal{P}$ denotes degree sum of all the non-incident edges to $u_{1}$ or $u_{2}$. If $e=\left(u_{1}, u_{2}\right)$ is deleted then

$$
\begin{gathered}
M_{2}(G-\{e\})=\left[d_{v_{1}} \cdot\left(d_{u_{1}}-1\right)+d_{v_{2}} \cdot\left(d_{u_{1}}-1\right)+\ldots+d_{v_{k}} \cdot\left(d_{u_{1}}-1\right)\right] \\
+\left[d_{w_{1}} \cdot\left(d_{u_{2}}-1\right)+d_{w_{2}} \cdot\left(d_{u_{2}}-1\right)+\ldots+d_{w_{l}} \cdot\left(d_{u_{2}}-1\right)\right]+\mathcal{P} \\
M_{2}(G-\{e\})-M_{2}(G)=-d_{u_{1}} \cdot d_{u_{2}}-\left[d_{v_{1}}-d_{v_{2}}-\ldots-d_{v_{k}}\right]-\left[d_{w_{1}}-d_{w_{2}}-\ldots-d_{w_{l}}\right] \\
M_{2}(G-\{e\})=M_{2}(G)-\sum_{u_{1} v_{i} \in E} d_{v_{i}}-\sum_{u_{2} w_{j} \in E} d_{w_{j}}-d_{u_{1}} \cdot d_{u_{2}}
\end{gathered}
$$


Mahesh Kale and Minirani S.

Theorem 3.3. Let $G$ be a simple graph with vertex set $\mathrm{V}$ and edge set $\mathrm{E}$, with $|V|=n$ and $|E|=m$. Let $e \in E$ be an edge incident to vertices $u_{1}$ and $u_{2}$ of the graph $G$, then the effect of edge deletion on the first multiplicative Zagreb index is given by

Proof: We have

$$
\frac{\Pi_{1}(G-\{e\})}{\Pi_{1}(G)}=\frac{\left[d_{u_{1}}-1\right]^{2} \cdot\left[d_{u_{2}}-1\right]^{2}}{d_{u_{1}}^{2} \cdot d_{u_{2}}^{2}}
$$

$$
\Pi_{1}(G)=\prod_{u \in V} d_{u}^{2}=d_{u_{1}}^{2} \cdot d_{u_{2}}^{2} \cdot d_{u_{3}}^{2} \ldots d_{u_{n}}^{2} .
$$

If an edge ' $e$ ' joining vertices $u_{1}$ and $u_{2}$ is deleted then their degrees decreases by 1 .

$$
\begin{gathered}
\Pi_{1}(G-\{e\})=\left(d_{u_{1}}-1\right)^{2} \cdot\left(d_{u_{2}}-1\right)^{2} \cdot d_{u_{3}}^{2} \ldots d_{u_{n}}^{2} \\
\frac{\Pi_{1}(G-\{e\})}{\Pi_{1}(G)}=\frac{\left[d_{u_{1}}-1\right]^{2} \cdot\left[d_{u_{2}}-1\right]^{2}}{d_{u_{1}}^{2} \cdot d_{u_{2}}^{2}}
\end{gathered}
$$

hence

Theorem 3.4. Let $G(V, E)$ be a simple graph. Let $e \in E$ be an edge incident to vertices $u_{1}$ and $u_{2}$ of the graph $G(V, E)$. Let $N\left(u_{1}\right)=\left\{u_{2}, v_{1}, v_{2}, \ldots, v_{k}\right\}$ and $N\left(u_{2}\right)=\left\{u_{1}, w_{1}, w_{2}, \ldots, w_{l}\right\}$ then the effect of edge deletion on the second multiplicative Zagreb index is given by

Proof: We have

$$
\frac{\Pi_{2}(G-\{e\})}{\Pi_{2}(G)}=\frac{\left(d_{u_{1}}-1\right)^{\left(d_{u_{1}}-1\right)} \cdot\left(d_{u_{2}}-1\right)^{\left(d_{u_{2}}-1\right)}}{d_{u_{1}}^{d_{u_{1}}} \cdot d_{u_{2}}^{d_{u_{2}}}}
$$

$$
\Pi_{2}(G)=\prod_{u \in V}\left(d_{u}\right)^{d_{u}}=d_{u_{1}}^{d_{u_{1}}} \cdot d_{u_{2}}^{d_{u_{2}}} \cdot d_{u_{3}}^{d_{u_{3}}} \ldots d_{u_{n}}^{d_{u_{n}}}
$$

If an edge adjoining vertices $u_{1}$ and $u_{2}$ is deleted then their degrees decreases by 1 .

hence

$$
\begin{gathered}
\Pi_{2}(G-\{e\})=\left(d_{u_{1}}-1\right)^{\left(d_{u_{1}}-1\right)} \cdot\left(d_{u_{2}}-1\right)^{\left(d_{u_{2}}-1\right)} \cdot d_{u_{3}}^{d_{u_{3}}} \ldots d_{u_{u_{n}}}^{d_{u_{n}}} \\
\frac{\Pi_{2}(G-\{e\})}{\Pi_{2}(G)}=\frac{\left(d_{u_{1}}-1\right)^{\left(d_{u_{1}}-1\right)} \cdot\left(d_{u_{2}}-1\right)^{\left(d_{u_{2}}-1\right)}}{d_{u_{1}}^{d_{u_{1}}} \cdot d_{u_{2}}^{d_{u_{2}}}}
\end{gathered}
$$

Theorem 3.5. Let $G(V, E)$ be a simple graph. Let $e \in E$ be an edge incident to vertices $u_{1}$ and $u_{2}$ of the graph $G(V, E)$. Let $N\left(u_{1}\right)=\left\{u_{2}, v_{1}, v_{2}, \ldots, v_{k}\right\}$ and $N\left(u_{2}\right)=\left\{u_{1}, w_{1}, w_{2}, \ldots, w_{l}\right\}$, then the effect of edge deletion on the multiplicative-sum Zagreb index is given by 
On Zagreb Indices of Graphs with a Deleted Edge

$$
\frac{\Pi_{1}^{*}(G-\{e\})}{\Pi_{1}^{*}(G)}=\prod_{i=1}^{k}\left[1-\frac{1}{d_{u_{1}}+d_{v_{i}}}\right] \cdot \prod_{j=1}^{l}\left[1-\frac{1}{d_{u_{2}}+d_{w_{j}}}\right] \cdot\left[\frac{1}{d_{u_{1}}+d_{u_{2}}}\right]
$$

Proof: By definition

$$
\begin{gathered}
\Pi_{1}^{*}(G)=\prod_{u v \in E}\left(d_{u}+d_{v}\right) \\
\Pi_{1}^{*}(G-\{e\})=\prod_{i=1}^{k}\left(d_{u_{1}}+d_{v_{i}}-1\right) \cdot \prod_{j=1}^{l}\left(d_{u_{2}}+d_{v_{j}}-1\right) \\
\frac{\Pi_{1}^{*}(G-\{e\})}{\Pi_{1}^{*}(G)}=\frac{U_{1} \cdot U_{2} \cdot \mathcal{P}}{U_{1}^{\prime} \cdot U_{2}^{\prime} \cdot\left[d_{u_{1}}+d_{u_{2}}\right] \cdot \mathcal{P}}
\end{gathered}
$$

where

$$
\begin{gathered}
U_{1}=\left(d_{u_{1}}+d_{v_{1}}-1\right)\left(d_{u_{1}}+d_{v_{2}}-1\right) \ldots\left(d_{u_{1}}+d_{v_{k}}-1\right) \\
U_{2}=\left(d_{u_{2}}+d_{w_{1}}-1\right)\left(d_{u_{2}}+d_{w_{2}}-1\right) \ldots\left(d_{u_{2}}+d_{w_{l}}-1\right) \\
U_{1}^{\prime}=\left(d_{u_{1}}+d_{v_{1}}\right)\left(d_{u_{1}}+d_{v_{2}}\right) \ldots\left(d_{u_{1}}+d_{v_{k}}\right) \\
U_{2}^{\prime}=\left(d_{u_{2}}+d_{w_{1}}\right)\left(d_{u_{2}}+d_{w_{2}}\right) \ldots\left(d_{u_{2}}+d_{w_{l}}\right)
\end{gathered}
$$

$\mathcal{P}$ denotes multiplicative degree sum of all the non-incident edges to $u_{1}$ or $u_{2}$.

$$
\begin{aligned}
\frac{\Pi_{1}^{*}(G-\{e\})}{\Pi_{1}^{*}(G)}= & \left(1-\frac{1}{d_{u_{1}}+d_{v_{1}}}\right)\left(1-\frac{1}{d_{u_{1}}+d_{v_{2}}}\right) \ldots\left(1-\frac{1}{d_{u_{1}}+d_{v_{k}}}\right) \\
& \cdot\left(1-\frac{1}{d_{u_{2}}+d_{w_{1}}}\right)\left(1-\frac{1}{d_{u_{2}}+d_{w_{2}}}\right) \ldots\left(1-\frac{1}{d_{u_{2}}+d_{w_{l}}}\right) \cdot\left(1-\frac{1}{d_{u_{1}}+d_{u_{2}}}\right) \\
= & \prod_{i=1}^{k}\left(1-\frac{1}{d_{u_{1}}+d_{v_{i}}}\right) \cdot \prod_{j=1}^{l}\left(1-\frac{1}{d_{u_{2}}+d_{w_{j}}}\right) \cdot\left(\frac{1}{d_{u_{1}}+d_{u_{2}}}\right)
\end{aligned}
$$

Theorem 3.6. Let $G(V, E)$ be a simple graph. $V=\left\{u_{1}, u_{2}, \ldots, u_{n}\right\}$. Let $e \in E$ be an edge incident to vertices $u_{1}$ and $u_{2}$ of the graph $G$, then the effect of edge deletion on the forgotten topological index is given by

$$
F(G-\{e\})=F(G)+3\left[d_{u_{1}}^{2}+d_{u_{2}}^{2}-d_{u_{1}}-d_{u_{2}}\right]+2
$$

Proof: By definition, first Zagreb index is given by

$$
F(G)=\sum_{u \in V} d_{u}^{3}=d_{u_{1}}^{3}+d_{u_{2}}^{3}+d_{u_{3}}^{3}+\ldots+d_{u_{n}}^{3}
$$

If $e=\left(u_{1}, u_{2}\right)$ is deleted then

$$
\begin{gathered}
F(G-\{e\})=\left(d_{u_{1}}-1\right)^{3}+\left(d_{u_{2}}-1\right)^{3}+d_{u_{3}}^{3}+\ldots+d_{u_{n}}^{3} \\
F(G-\{e\})-F(G)=\left(d_{u_{1}}-1\right)^{3}+\left(d_{u_{2}}-1\right)^{3}-d_{u_{1}}^{3}-d_{u_{2}}^{3}
\end{gathered}
$$


Mahesh Kale and Minirani S.

$$
F(G-\{e\})=F(G)-3\left(d_{u_{1}}^{2}+d_{u_{2}}^{2}-d_{u_{1}}-d_{u_{2}}\right)-2
$$

Theorem 3.7. Let $G$ be a simple graph with vertex set $V$ and edge set $E$, with $|V|=n$ and $|E|=m$. Let $e \in E$ be an edge incident to vertices $u_{1}$ and $u_{2}$ of graph $G$. Let $N\left(u_{1}\right)=\left\{u_{2}, v_{1}, v_{2}, \ldots, v_{k}\right\}$ and $N\left(u_{2}\right)=\left\{u_{1}, w_{1}, w_{2}, \ldots, w_{l}\right\}$, where $k+l+2 \leq n$, then the effect of edge deletion on the Hyper Zagreb index is given by

$$
\begin{aligned}
H M(G-\{e\})= & H M(G)+3\left[d_{u_{1}}+d_{u_{2}}\right]-2\left[d_{u_{1}}^{2}+d_{u_{2}}^{2}\right]-\left[d_{u_{1}}+d_{u_{2}}\right]^{2} \\
& -2 \sum_{i=1}^{k} d_{v_{i}}-2 \sum_{j=1}^{l} d_{w_{j}}-2
\end{aligned}
$$

Proof: By definition, hyper Zagreb index is given as

$$
\begin{gathered}
H M(G)=\sum_{u v \in E}\left(d_{u}+d_{v}\right)^{2} \\
H M(G-\{e\})=\left[\left(d_{u_{1}}+d_{v_{1}}-1\right)^{2}+\left(d_{u_{1}}+d_{v_{2}}-1\right)^{2}+\ldots+\left(d_{u_{1}}+d_{v_{k}}-1\right)^{2}\right] \\
+\left[\left(d_{u_{2}}+d_{w_{1}}-1\right)^{2}+\left(d_{u_{2}}+d_{w_{2}}-1\right)^{2}+\ldots+\left(d_{u_{2}}+d_{w_{l}}-1\right)^{2}\right]+\mathcal{P}
\end{gathered}
$$

where $\mathcal{P}$ denotes sum of square of degree sum of all the non-incident edges to $u_{1}$ or $u_{2}$. Hence

$$
\begin{aligned}
H M(G-\{e\})-H M(G)= & \sum_{i=1}^{k}\left[d_{v_{i}}+d_{u_{1}}-1\right]^{2}+\sum_{j=1}^{l}\left[d_{w_{j}}+d_{u_{2}}-1\right]^{2}-\left[d_{u_{1}}+d_{u_{2}}\right]^{2} \\
& -\sum_{i=1}^{k}\left[d_{v_{i}}+d_{u_{1}}\right]^{2}+\sum_{j=1}^{l}\left[d_{w_{j}}+d_{u_{2}}\right]^{2} \\
H M(G-\{e\})= & H M(G)+3\left[d_{u_{1}}+d_{u_{2}}\right]-2\left[d_{u_{1}}^{2}+d_{u_{2}}^{2}\right]-\left[d_{u_{1}}+d_{u_{2}}\right]^{2} \\
& -2 \sum_{i=1}^{k} d_{v_{i}}-2 \sum_{j=1}^{l} d_{w_{j}}-2
\end{aligned}
$$

Theorem 3.8. Let $G(V, E)$ be a simple graph. Let $e \in E$ be an edge incident to vertices $u_{1}$ and $u_{2}$ of graph $G(V, E)$. Let $N\left(u_{1}\right)=\left\{u_{2}, v_{1}, v_{2}, \ldots, v_{k}\right\}$ and $N\left(u_{2}\right)=\left\{u_{1}, w_{1}, w_{2}, \ldots, w_{l}\right\}$, then the effect of edge deletion on the first multiplicative hyper Zagreb index is given by

$$
\frac{H \Pi_{1}(G-\{e\})}{H \Pi_{1}(G)}=\prod_{i=1}^{k}\left(1-\frac{1}{d_{u_{1}}+d_{v_{i}}}\right)^{2} \cdot \prod_{j=1}^{l}\left(1-\frac{1}{d_{u_{2}}+d_{w_{j}}}\right)^{2} \cdot\left(\frac{1}{d_{u_{1}}+d_{u_{2}}}\right)^{2}
$$

Proof: By definition first multiplicative hyper Zagreb index is defined as

$$
H \Pi_{1}(G)=\prod_{u v E}\left(d_{u}+d_{v}\right)^{2}
$$


On Zagreb Indices of Graphs with a Deleted Edge

$$
\begin{gathered}
H \Pi_{1}(G-\{e\})=\prod_{i=1}^{k}\left(d_{u_{1}}+d_{v_{i}}-1\right)^{2} \cdot \prod_{j=1}^{l}\left(d_{u_{2}}+d_{v_{j}}-1\right)^{2} \\
\frac{H \Pi_{1}(G-\{e\})}{H \Pi_{1}(G)}=\frac{U_{1} \cdot U_{2} \cdot \mathcal{P}}{U_{1}^{\prime} \cdot U_{2}^{\prime} \cdot\left[d_{u_{1}}+d_{u_{2}}\right]^{2} \cdot \mathcal{P}}
\end{gathered}
$$

where

$$
\begin{gathered}
U_{1}=\left(d_{u_{1}}+d_{v_{1}}-1\right)^{2}\left(d_{u_{1}}+d_{v_{2}}-1\right)^{2} \ldots\left(d_{u_{1}}+d_{v_{k}}-1\right)^{2} \\
U_{2}=\left(d_{u_{2}}+d_{w_{1}}-1\right)^{2}\left(d_{u_{2}}+d_{w_{2}}-1\right)^{2} \ldots\left(d_{u_{2}}+d_{w_{l}}-1\right)^{2} \\
U_{1}^{\prime}=\left(d_{u_{1}}+d_{v_{1}}\right)^{2}\left(d_{u_{1}}+d_{v_{2}}\right)^{2} \ldots\left(d_{u_{1}}+d_{v_{k}}\right)^{2} \\
U_{2}^{\prime}=\left(d_{u_{2}}+d_{w_{1}}\right)^{2}\left(d_{u_{2}}+d_{w_{2}}\right)^{2} \ldots\left(d_{u_{2}}+d_{w_{l}}\right)^{2}
\end{gathered}
$$

$\mathcal{P}$ denotes multiplicative degree sum of all the non-incident edges to $u_{1}$ or $u_{2}$.

$$
\begin{aligned}
& \frac{H \Pi_{1}(G-\{e\})}{H \Pi_{1}(G)=}\left(1-\frac{1}{d_{u_{1}}+d_{v_{1}}}\right)^{2}\left(1-\frac{1}{d_{u_{1}}+d_{v_{2}}}\right)^{2} \ldots\left(1-\frac{1}{d_{u_{1}}+d_{v_{k}}}\right)^{2} \\
& \cdot\left(1-\frac{1}{d_{u_{2}}+d_{w_{1}}}\right)^{2}\left(1-\frac{1}{d_{u_{2}}+d_{w_{2}}}\right)^{2} \ldots\left(1-\frac{1}{d_{u_{2}}+d_{w_{l}}}\right)^{2} . \\
& \cdot\left(1-\frac{1}{d_{u_{1}}+d_{u_{2}}}\right)^{2} \\
&= \prod_{i=1}^{k}\left(1-\frac{1}{d_{u_{1}}+d_{v_{i}}}\right)^{2} \cdot \prod_{j=1}^{l}\left(1-\frac{1}{d_{u_{2}}+d_{w_{j}}}\right)^{2} \cdot\left(\frac{1}{d_{u_{1}}+d_{u_{2}}}\right)^{2}
\end{aligned}
$$

Theorem 3.9. Let $G(V, E)$ be a simple graph. Let $e \in E$ be an edge incident to vertices $u_{1}$ and $u_{2}$ of the graph $G(V, E)$. Let $N\left(u_{1}\right)=\left\{u_{2}, v_{1}, v_{2}, \ldots, v_{k}\right\}$ and $N\left(u_{2}\right)=\left\{u_{1}, w_{1}, w_{2}, \ldots, w_{l}\right\}$, then the effect of edge deletion on the second multiplicative hyper Zagreb index is given by

$$
\frac{H \Pi_{2}(G-\{e\})}{H \Pi_{2}(G)}=\frac{\left(d_{u_{1}}-1\right)^{2\left(d_{u_{1}}-1\right)} \cdot\left(d_{u_{2}}-1\right)^{2\left(d_{u_{2}}-1\right)}}{d_{u_{1}}^{2 d_{u_{1}}} \cdot d_{u_{2}}^{2 d_{u_{2}}}}
$$

Proof: By definition, second multiplicative hyper Zagreb index is defined as

$$
\begin{gathered}
H \Pi_{2}(G)=\prod_{u v E E}\left(d_{u} \cdot d_{v}\right)^{2} \\
H \Pi_{2}(G-\{e\})=\prod_{i=1}^{k}\left[d_{v_{i}}^{2} \cdot\left(d_{u_{1}}-1\right)^{2}\right] \cdot \prod_{j=1}^{l}\left[d_{w_{j}}^{2} \cdot\left(d_{u_{2}}-1\right)^{2}\right] \cdot \mathcal{P}
\end{gathered}
$$


Mahesh Kale and Minirani S.

where $\mathcal{P}$ denotes multiplicative degree sum of all the non-incident edges to $u_{1}$ or $u_{2}$.

$$
\frac{H \Pi_{2}(G-\{e\})}{H \Pi_{2}(G)}=\frac{U_{1} \cdot U_{2} \cdot \mathcal{P}}{U_{1}^{\prime} \cdot U_{2}^{\prime} \cdot\left[d_{u_{1}} \cdot d_{u_{2}}\right]^{2} \cdot \mathcal{P}}
$$

where,

$$
\begin{gathered}
U_{1}=d_{v_{1}}^{2}\left(d_{u_{1}}-1\right)^{2} \cdot d_{v_{2}}^{2}\left(d_{u_{1}}-1\right)^{2} \ldots d_{v_{k}}^{2}\left(d_{u_{1}}-1\right)^{2} \\
U_{2}=d_{w_{1}}^{2}\left(d_{u_{2}}-1\right)^{2} \cdot d_{w_{2}}^{2}\left(d_{u_{2}}-1\right)^{2} \ldots d_{w_{l}}^{2}\left(d_{u_{2}}-1\right)^{2} \\
U_{1}^{\prime}=\left(d_{u_{1}} \cdot d_{v_{1}}\right)^{2} \cdot\left(d_{u_{1}} \cdot d_{v_{2}}\right)^{2} \ldots\left(d_{u_{1}} \cdot d_{v_{k}}\right)^{2} \\
U_{2}^{\prime}=\left(d_{u_{2}} \cdot d_{w_{1}}\right)^{2} \cdot\left(d_{u_{2}} \cdot d_{w_{2}}\right)^{2} \ldots\left(d_{u_{2}} \cdot d_{w_{l}}\right)^{2}
\end{gathered}
$$

$\mathcal{P}$ denotes multiplicative degree sum of all the non-incident edges to $u_{1}$ or $u_{2}$.

$$
\begin{aligned}
\frac{H \Pi_{2}(G-\{e\})}{H \Pi_{2}(G)} & =\frac{\left(d_{u_{1}}-1\right)^{2 k} \cdot\left(d_{u_{2}}-1\right)^{2 l}}{\left(d_{u_{1}}\right)^{2 k} \cdot\left(d_{u_{2}}\right)^{2 l} \cdot\left[d_{u_{1}} \cdot d_{u_{2}}\right]^{2}} \\
& =\frac{\left(d_{u_{1}}-1\right)^{2\left(d_{u_{1}}-1\right)} \cdot\left(d_{u_{2}}-1\right)^{2\left(d_{u_{2}}-1\right)}}{d_{u_{1}}^{2 d_{u_{1}}} \cdot d_{u_{2}}^{2 d_{u_{2}}}}
\end{aligned}
$$

\section{Deleting an edge from some standard graphs}

Here we discuss the effect on Zagreb indices after deletion an edge from some standard graphs such as $P_{n}, C_{n}, S_{n}, K_{n}$ and $K_{m, n}$ and verify the effect using the results obtained. Let $e=(i, j)$ denotes an edge joining end vertices with degrees ' $i$ ' and ' $j$ '.

\subsection{Path graph $P_{n}$}

In case of path graph ' $P_{n}{ }^{\prime} ; n \geq 4$, there are two types of edges $e_{1}(2,1)$ and $e_{2}(2,2)$

i) $\quad M_{1}\left(P_{n}\right)=4 n-6$

$$
\begin{aligned}
& M_{1}\left(P_{n}-\left\{e_{1}\right\}\right)=4 n-10=(4 n-6)-2(2+1-1) \\
& M_{1}\left(P_{n}-\left\{e_{2}\right\}\right)=4 n-12=(4 n-6)-2(2+2-1)
\end{aligned}
$$

ii) $\quad M_{2}\left(P_{n}\right)=4 n-8$

$$
\begin{aligned}
& M_{2}\left(P_{n}-\left\{e_{1}\right\}\right)=4 n-12=(4 n-8)-2-0-2 \\
& M_{2}\left(P_{n}-\left\{e_{2}\right\}\right)=4 n-16=(4 n-8)-2-2-4
\end{aligned}
$$

iii) $\Pi_{1}\left(P_{n}\right)=(2)^{2(n-2)}$

$$
\Pi_{1}\left(P_{n}-\left\{e_{1}\right\}\right)=0
$$

$\Pi_{1}\left(P_{n}-\left\{e_{2}\right\}\right)=(2)^{2(n-4)}=\frac{1}{2^{2} \cdot 2^{2}} \cdot(2)^{2(n-2)}$

iv) $\Pi_{2}\left(P_{n}\right)=(2)^{2(n-2)}$

$$
\Pi_{2}\left(P_{n}-\left\{e_{1}\right\}\right)=0
$$


On Zagreb Indices of Graphs with a Deleted Edge

$$
\Pi_{2}\left(P_{n}-\left\{e_{2}\right\}\right)=(2)^{2(n-4)}=\frac{1}{2^{2} \cdot 2^{2}} \cdot(2)^{2(n-2)}
$$

v) $\Pi_{1}^{*}\left(P_{n}\right)=3^{2} \cdot(2)^{2(n-3)}$

$$
\begin{aligned}
& \Pi_{1}^{*}\left(P_{n}-\left\{e_{1}\right\}\right)=3^{2} \cdot(2)^{2(n-4)}=\frac{3}{4} \cdot \frac{3^{2}}{3} \cdot(2)^{2(n-3)} \\
& \Pi_{1}^{*}\left(P_{n}-\left\{e_{2}\right\}\right)=3^{4} \cdot(2)^{2(n-6)}=\frac{3}{4} \cdot \frac{3}{4} \cdot \frac{3^{2}}{4} \cdot(2)^{2(n-3)}
\end{aligned}
$$

vi) $F\left(P_{n}\right)=8 n-22$

$$
\begin{aligned}
& F\left(P_{n}-\left\{e_{1}\right\}\right)=8 n-30=(8 n-22)-3\left(2^{2}+1-2-1\right)-2 \\
& F\left(P_{n}-\left\{e_{2}\right\}\right)=8 n-36=(8 n-22)-3\left(2^{2}+2^{2}-2-2\right)-2
\end{aligned}
$$

vii) $H M\left(P_{n}\right)=16 n-30$

$$
\begin{aligned}
& H M\left(P_{n}-\left\{e_{1}\right\}\right)=16 n-46=(16 n-30)+3(2+1)-2(4+1)-6-(2+1)^{2} \\
& H M\left(P_{n}-\left\{e_{2}\right\}\right)=16 n-60=(16 n-30)+3(4)-2(8)-(4)^{2}-8-2
\end{aligned}
$$

viii $H \Pi_{1}\left(P_{n}\right)=3^{4} \cdot(4)^{2(n-3)}$

$$
\begin{aligned}
& H \Pi_{1}\left(P_{n}-\left\{e_{1}\right\}\right)=3^{4} \cdot(4)^{2(n-4)}=3^{4} \cdot(4)^{2(n-3)} \cdot \frac{1}{9} \cdot \frac{9}{16} \\
& H \Pi_{1}\left(P_{n}-\left\{e_{2}\right\}\right)=3^{8} \cdot(4)^{2(n-6)}=3^{4} \cdot(4)^{2(n-3)} \cdot \frac{1}{16} \cdot \frac{9}{16} \cdot \frac{9}{16}
\end{aligned}
$$

ix) $H \Pi_{2}\left(P_{n}\right)=(4)^{2(n-2)}$

$$
H \Pi_{2}\left(P_{n}-\left\{e_{1}\right\}\right)=0
$$

$$
H \Pi_{2}\left(P_{n}-\left\{e_{2}\right\}\right)=(4)^{2(n-4)}=(4)^{2(n-2)} \cdot \frac{1}{2^{4} \cdot 2^{4}}
$$

\subsection{Cycle graph $C_{n}$}

In case of cycle graph ' $C_{n}$ ', any edge is of type $e(2,2)$

$$
\begin{array}{ll}
\text { i) } & M_{1}\left(C_{n}\right)=4 n \\
& M_{1}\left(C_{n}-\{e\}\right)=4 n-6=4 n-2(2+2-1) \\
\text { ii) } & M_{2}\left(C_{n}\right)=4 n \\
& M_{2}\left(C_{n}-\{e\}\right)=4 n-8=4 n-2-2-4 \\
\text { iii) } & \Pi_{1}\left(C_{n}\right)=(2)^{2 n} \\
& \Pi_{1}\left(C_{n}-\{e\}\right)=(2)^{2(n-2)}=2^{2 n} \cdot \frac{1}{2^{2} \cdot 2^{2}} \\
\text { iv) } & \Pi_{2}\left(C_{n}\right)=(2)^{2 n}
\end{array}
$$


Mahesh Kale and Minirani S.

$$
\begin{array}{ll} 
& \Pi_{2}\left(C_{n}-\{e\}\right)=(2)^{2(n-2)}=2^{2 n} \cdot \frac{1}{2^{2} \cdot 2^{2}} \\
\text { v) } \quad \Pi_{1}^{*}\left(C_{n}\right)=(2)^{2 n} \\
\\
\Pi_{1}^{*}\left(C_{n}-\{e\}\right)=3^{2} \cdot(2)^{2(n-3)}=2^{2 n} \cdot \frac{3}{4} \cdot \frac{3}{4} \cdot \frac{1}{4} \\
\text { vi) } & F\left(C_{n}\right)=8 n \\
& F\left(C_{n}-\{e\}\right)=8 n-14=8 n-3(4+4-2-2)-2 \\
\text { vii) } \quad H M\left(C_{n}\right)=16 n \\
\text { viii) } & H M\left(C_{n}-\{e\}\right)=16 n-30=16 n+3(2+2)-2(8)-4^{2}-4-4-2 \\
& H \Pi_{1}\left(C_{n}-\{e\}\right)=3^{4} \cdot(4)^{2(n-3)}=4^{2 n} \cdot \frac{9}{16} \cdot \frac{9}{16} \cdot \frac{1}{16} \\
\text { ix) } & H \Pi_{2}\left(C_{n}\right)=(4)^{2 n} \\
& H \Pi_{2}\left(C_{n}-\{e\}\right)=(4)^{2(n-2)}=4^{2 n} \cdot \frac{1}{16} \cdot \frac{1}{16}
\end{array}
$$

\subsection{Star graph $S_{n}$}

In case of star graph ' $S_{n}$ ' with $n(\geq 4)$, any edge is of type $e(n-1,1)$

$$
\begin{array}{ll}
\text { i) } & M_{1}\left(S_{n}\right)=n(n-1) \\
& M_{1}\left(S_{n}-\{e\}\right)=(n-1)(n-2)=n(n-1)-2(n-1) \\
& M_{2}\left(S_{n}\right)=(n-1)^{2} \\
& M_{2}\left(S_{n}-\{e\}\right)=(n-2)^{2}=(n-1)^{2}-(n-2)-(n-1) \\
\text { iii) } & \Pi_{1}\left(S_{n}\right)=(n-1)^{2} \\
& \Pi_{1}\left(S_{n}-\{e\}\right)=0=(n-1)^{2} .0 \\
\text { iv) } & \Pi_{2}\left(S_{n}\right)=(n-1)^{(n-1)} \\
& \Pi_{2}\left(S_{n}-\{e\}\right)=0=(n-1)^{(n-1)} \cdot 0 \\
& \Pi_{1}^{*}\left(S_{n}\right)=(n)^{(n-1)} \\
& \Pi_{1}^{*}\left(S_{n}-\{e\}\right)=(n-1)^{(n-2)}=n^{(n-1)} \cdot\left(1-\frac{1}{n}\right)^{(n-2)} \cdot\left(\frac{1}{n}\right) \\
\text { vi) } & F\left(S_{n}\right)=(n-1)+(n-1)^{3} \\
& F\left(S_{n}-\{e\}\right)=(n-2)+(n-2)^{3} \\
& \quad=(n-1)+(n-1)^{3}-3\left[(n-1)^{2}-(n-1)\right]-2 \\
\text { vii) } & H M\left(S_{n}\right)=n^{2}(n-1)
\end{array}
$$


On Zagreb Indices of Graphs with a Deleted Edge

$$
\begin{aligned}
H M\left(S_{n}-\{e\}\right) & =(n-1)^{2}(n-2) \\
& =n^{2}(n-1)+3 n-2\left[(n-1)^{2}+1\right]-n^{2}-2(n-2)-2
\end{aligned}
$$

viii) $H \Pi_{1}\left(S_{n}\right)=n^{2(n-1)}$

ix) $\quad H \Pi_{2}\left(S_{n}\right)=(n-1)^{2(n-1)}$

$$
H \Pi_{1}\left(S_{n}-\{e\}\right)=(n-1)^{2(n-2)}=n^{2(n-1)} \cdot\left(1-\frac{1}{n}\right)^{2(n-2)} \cdot \frac{1}{n^{2}}
$$

$$
H \Pi_{2}\left(S_{n}-\{e\}\right)=(n-2)^{2(n-2)}=(n-1)^{2(n-1)} \cdot \frac{(n-2)^{2(n-2)}}{(n-1)^{2(n-1)}}
$$

\subsection{Complete graph $K_{n}$}

In case of complete graph ' $K_{n}$ ', any edge is of type $e(n-1, n-1)$

i) $\quad M_{1}\left(K_{n}\right)=n(n-1)^{2}$

$$
M_{1}\left(K_{n}-\{e\}\right)=n^{3}-2 n^{2}-3 n+6=n(n-1)^{2}-2(2 n-3)
$$

ii)

$$
\begin{aligned}
& M_{2}\left(K_{n}\right)=\frac{n(n-1)^{3}}{2} \\
& M_{2}\left(K_{n}-\{e\}\right)=\frac{(n-1)(n-2)\left(n^{2}-5\right)}{2}=\frac{n(n-1)^{3}}{2}-2(n-1)(n-2)-(n-1)^{2}
\end{aligned}
$$

iii) $\Pi_{1}\left(K_{n}\right)=(n-1)^{2 n}$

$$
\Pi_{1}\left(K_{n}-\{e\}\right)=(n-1)^{2(n-2)} \cdot(n-2)^{4}=(n-1)^{2 n} \cdot \frac{(n-2)^{4}}{(n-1)^{4}}
$$

iv) $\quad \Pi_{2}\left(K_{n}\right)=(n-1)^{n(n-1)}$

$$
\Pi_{2}\left(K_{n}-\{e\}\right)=(n-1)^{(n-1)(n-2)} \cdot(n-2)^{2(n-2)}=(n-1)^{n(n-1)} \cdot \frac{(n-2)^{2(n-2)}}{(n-1)^{2(n-1)}}
$$

v) $\quad \Pi_{1}^{*}=(2 n-2)^{n(n-1) / 2}$

$$
\begin{aligned}
\Pi_{1}^{*}\left(K_{n}-\{e\}\right) & =(2 n-2)^{(n-2)(n-3) / 2} \cdot(2 n-3)^{2(n-2)} \\
& =(2 n-2)^{n(n-1) / 2} \cdot\left(\frac{2 n-3}{2 n-2}\right)^{2(n-2)} \cdot\left(\frac{1}{2 n-2}\right)
\end{aligned}
$$

vi) $\quad F\left(K_{n}\right)=n(n-1)^{3}$

$$
F\left(K_{n}-\{e\}\right)=(n-1)^{3}(n-2)+2(n-2)^{3}=n(n-1)^{3}-6\left[(n-1)^{2}-(n-1)\right]-2
$$

vii) $H M\left(K_{n}\right)=2 n(n-1)^{3}$

$$
\begin{aligned}
H M\left(K_{n}-\{e\}\right) & =2\left(n^{4}-3 n^{3}-3 n^{2}+14 n-10\right) \\
& =2 n(n-1)^{3}+6(n-1)-12(n-1)^{2}-2
\end{aligned}
$$


Mahesh Kale and Minirani S.

viii) $H \Pi_{1}\left(K_{n}\right)=(2 n-2)^{n(n-1)}$

$$
\begin{aligned}
H \Pi_{1}\left(K_{n}-\{e\}\right) & =(2 n-3)^{4(n-2)} \cdot(2 n-2)^{\left(n^{2}-5 n+6\right)} \\
& =(2 n-2)^{n(n-1)} \cdot\left(\frac{2 n-3}{2 n-2}\right)^{4(n-2)} \cdot\left(\frac{1}{2 n-2}\right)^{2}
\end{aligned}
$$

ix) $\quad H \Pi_{2}\left(K_{n}\right)=(n-1)^{2 n(n-1)}$

$$
H \Pi_{2}\left(K_{n}-\{e\}\right)=(n-1)^{2(n-1)(n-2)} \cdot(n-2)^{4(n-2)}=(n-1)^{2 n(n-1)} \cdot \frac{(n-2)^{4(n-2)}}{(n-1)^{4(n-1)}}
$$

\subsection{Complete bipartite graph $K_{m, n}$}

In case of complete bipartite graph ' $K_{m, n}$ '; with $m, n \geq 2$, any edge is of type $e(n, m)$

i) $\quad M_{1}\left(K_{m, n}\right)=(m n) \cdot(m+n)$

$$
M_{1}\left(K_{m, n}-\{e\}\right)=(m+n) .(m n-2)+2=m n .(m+n)-2(m+n-1)
$$

ii) $\quad M_{2}\left(K_{m, n}\right)=m^{2} \cdot n^{2}$

$$
M_{2}\left(K_{m, n}-\{e\}\right)=m^{2} n^{2}-3 m n+m+n=m^{2} n^{2}-m(n-1)-n(m-1)-m n
$$

iii) $\quad \Pi_{1}\left(K_{m, n}\right)=n^{2 m} \cdot m^{2 n}$

$$
\begin{aligned}
\Pi_{1}\left(K_{m, n}-\{e\}\right) & =(m-1)^{2} \cdot(n-1)^{2} \cdot m^{2(n-1)} \cdot n^{2(m-1)} \\
& =n^{2 m} \cdot m^{2 n} \cdot \frac{(n-1)^{2} \cdot(m-1)^{2}}{m^{2} \cdot n^{2}}
\end{aligned}
$$

iv) $\quad \Pi_{2}\left(K_{m, n}\right)=n^{m n} \cdot m^{m n}$

$$
\begin{aligned}
\Pi_{2}\left(K_{m, n}-\{e\}\right) & =(m-1)^{(m-1)} \cdot(n-1)^{(n-1)} \cdot m^{m(n-1)} \cdot n^{n(m-1)} \\
& =n^{m n} \cdot m^{n m} \cdot \frac{(m-1)^{(m-1)} \cdot(n-1)^{(n-1)}}{m^{m} \cdot n^{n}}
\end{aligned}
$$

v) $\quad \Pi_{1}^{*}\left(K_{m, n}\right)=(m+n)^{(m n)}$

$$
\begin{aligned}
\Pi_{1}^{*}\left(K_{m, n}-\{e\}\right) & =(m+n-1)^{(m+n-2)} \cdot(m+n)^{(m n-m-n+1)} \\
& =(m+n)^{m n} \cdot \frac{(m+n-1)^{(n-1)} \cdot(m+n-1)^{(m-1)}}{(m+n)^{(m+n-1)}}
\end{aligned}
$$

vi) $\quad F\left(K_{m, n}\right)=m \cdot n^{3}+n \cdot m^{3}$

$$
\begin{aligned}
F\left(K_{m, n}-\{e\}\right) & =(m-1)^{3}+(n-1)^{3}+m^{3}(n-1)+n^{3}(m-1) \\
& =m \cdot n^{3}+n \cdot m^{3}-3\left(m^{2}+n^{2}-m-n\right)-2
\end{aligned}
$$

vii) $\quad H M\left(K_{m, n}\right)=(m n)(m+n)^{2}$ 
On Zagreb Indices of Graphs with a Deleted Edge

$$
\begin{aligned}
& H M\left(K_{m, n}-\{e\}\right)=(m+n-1)^{2}(m+n-2)+(m+n)^{2}(m n-n-m+1) \\
& =m n \cdot(m+n)^{2}+3(m+n)-2\left(m^{2}+n^{2}\right) \\
& -(m+n)^{2}-2 m(n-1)-2 n(m-1)-2 \\
& \text { viii) } H \Pi_{1}\left(K_{m, n}\right)=(m+n)^{2(m n)} \\
& H \Pi_{1}\left(K_{m, n}-\{e\}\right)=(m+n-1)^{2(m+n-2)} \cdot(m+n)^{2(m n-n-m+1)} \\
& =(m+n)^{2 m n} \cdot\left(\frac{m+n-1}{m+n}\right)^{2(n-1)} \\
& \cdot\left(\frac{m+n-1}{m+n}\right)^{2(m-1)} \cdot \frac{1}{(m+n)^{2}} \\
& H \Pi_{2}\left(K_{m, n}-\{e\}\right)=[(m-1) n]^{2(m-1)} \cdot[m(n-1)]^{2(n-1)}\left(m^{2} n^{2}\right) \cdot(m n-n-m+1) \\
& =(m n)^{2 m n} \cdot \frac{(n-1)^{2(n-1)} \cdot(m-1)^{2(m-1)}}{n^{2 n} \cdot m^{2 m}}
\end{aligned}
$$

\section{Conclusion}

We have studied the properties of Zagreb indices and the effect on them when an edge is deleted from a given graph. We have also verified the results obtained with some of the standard graphs by taking into consideration all the possible cases. More properties and applications of Zagreb indices will be discussed in the following papers.

Acknowledgements. The authors are highly grateful to the anonymous reviewers for their helpful comments and suggestions for improving the paper.

\section{REFERENCES}

1. I.Gutman and N.Trinajsti'c, Graph theory and molecular orbitals. total $\phi$-electron energy of alternant hydrocarbons, Chemical Physics Letters, 17 (1972) 535-538.

2. M.Azari and A.Iranmanesh, Chemical graphs constructed from rooted product and their zagreb indices, MATCH Commun. Math. Comput. Chem, 70 (2013) 901-919.

3. V.Kulli, On reduced zagreb indices of polycyclic aromatic hydrocarbons and benzenoid systems, Annals of Pure and Applied Mathematics, 18 (2018) 73-78.

4. Khalifeh, H.Y.-Azari, and A.R.Ashrafi, The first and second Zagreb indices of some graph operations, Discrete Applied Mathematics, 157 (2009) 804-811.

5. V.Kulli, Reverse Zagreb and reverse hyper-Zagreb indices and their polynomials of rhombus silicate networks, Annals of Pure and Applied Mathematics, 16 (2018) 4751.

6. S.Nikoli'c, G.Kova ̌cevi'c, A.Milicevi'c, and N.Trinajsti'c, The zagreb indices 30 years after, Croatica Chemica Acta, 76 (2003) 113-124.

7. B.Zhou and I.Gutman, Further properties of zagreb indices, MATCH Commun. Math. Comput. Chem, 54 (2005) 233-239. 
Mahesh Kale and Minirani S.

8. M.Azari, Sharp lower bounds on the narumi-katayama index of graph operations, Applied Mathematics and Computation, 239 (2014) 409-421.

9. M.Azari and A.Iranmanesh, Some inequalities for the multiplicative sum zagreb index of graph operations, J. Math. Inequal, 9 (2015) 727- 738.

10. M.Eliasi, A simple approach to order the multiplicative zagreb indices of connected graphs, Trans. Comb, 1 (2012) 17-24.

11. M.Eliasi and D.Vukicevic, Comparing the multiplicative zagreb indices, MATCH Commun. Math. Comput. Chem, 69 (2013) 765-773.

12. F.F.Nezhad, A.Iranmanesh, A.Tehranian and M.Azari, Strict lower bounds on the multiplicative zagreb indices of graph operations, ARS Combinatoria, 117 (2014) 399-409.

13. T.Reti and I.Gutman, Relations between ordinary and multiplicative zagreb indices, Bull. Internat. Math. Virt. Inst, 2 (2012) 133-140.

14. K.Xu and H.Hua, A unified approach to extremal multiplicative zagreb indices for trees, unicyclic and bicyclic graphs, Match-Communications in Mathematical and Computer Chemistry, 68 (2012) 241.

15. B.Furtula and I.Gutman, A forgotten topological index, Journal of Mathematical Chemistry, 53 (2015) 1184-1190.

16. M.Binu, S.Mathew and J.Mordeson, Wiener index of a fuzzy graph and application to illegal immigration networks, Fuzzy Sets and Systems, 384 (2020) 132-147.

17. R.Islam, S.Maity and M.Pal, Comment on "Wiener index of a fuzzy graph and application to illegal immigration networks", Fuzzy Sets and Systems, 384 (2020) 148- 151.

18. R.Todeschini and V.Consonni, New local vertex invariants and molecular descriptors based on functions of the vertex degrees, MATCH Commun. Math. Comput. Chem, 64 (2010) 359-372.

19. M.Eliasi, A.Iranmanesh, and I.Gutman, Multiplicative versions of first zagreb index, Match-Communications in Mathematical and Computer Chemistry, 68 (2012) 217.

20. I.Gutman, Multiplicative zagreb indices of trees, Bull. Int. Math. Virt. Inst, 1 (2011) $13-19$.

21. G.Shirdel, H.Rezapour, and A.Sayadi, The hyper-zagreb index of graph operations, Iranian Journal of Mathematical Chemistry, 4 (2013) 213-220.

22. V.Kulli, Multiplicative connectivity indices of certain nanotubes, Annals of Pure and Applied Mathematics, 12 (2016) 169-176. 